\title{
Behavioral and Physiological Responses of Ozark Zigzag Salamanders to Stimuli from an Invasive Predator: The Armadillo
}

\author{
Adam L. Crane, Carly E. McGrane, and Alicia Mathis \\ Department of Biology, Missouri State University, 901 S. National, Springfield, MO 65897, USA
}

Correspondence should be addressed to Adam L. Crane, adamcrane@missouristate.edu

Received 8 July 2011; Accepted 18 August 2011

Academic Editor: Andrew Sih

Copyright (C) 2012 Adam L. Crane et al. This is an open access article distributed under the Creative Commons Attribution License, which permits unrestricted use, distribution, and reproduction in any medium, provided the original work is properly cited.

\begin{abstract}
When new predators invade a habitat, either through range extensions or introductions, prey may be at a high risk because they do not recognize the predators as dangerous. The nine-banded armadillo (Dasypus novemcinctus) has recently expanded its range in North America. Armadillos forage by searching soil and leaf litter, consuming invertebrates and small vertebrates, including salamanders. We tested whether Ozark zigzag salamanders (Plethodon angusticlavius) from a population coexisting with armadillos for about 30 years exhibit antipredator behavior in the presence of armadillo chemical cues and whether they can discriminate between stimuli from armadillos and a nonpredatory sympatric mammal (white-tailed deer, Odocoileus virginianus). Salamanders appeared to recognize substrate cues from armadillos as a threat because they increased escape behaviors and oxygen consumption. When exposed to airborne cues from armadillos, salamanders also exhibited an antipredator response by spending more time in an inconspicuous posture. Additionally, individually consistent behaviors across treatments for some response variables suggest the potential for a behavioral syndrome in this species.
\end{abstract}

\section{Introduction}

Invasive species have been implicated as a factor in population declines [1] and extinctions [2] of many species. Whether due to introductions or range expansions, invasive species can present challenges to native fauna in the form of competition [3], predation [1], or introductions of disease or parasites [4]. These problems can be exacerbated by the inability of native species to recognize the threat imposed by invaders so that they fail to perform appropriate evasive or protective behaviors (e.g., [5-7]).

Predicting how prey respond to introduced predators is not always straight forward. On the one hand, prey may fail to respond appropriately to novel predators due to lack of recognition of danger. On the other hand, introduced predators may sometimes be less of a problem to native prey. For example, in some cases, prey recognize cues from introduced predators without experience, possibly because the cues are similar to cues from their native predators ("generalized predator recognition:" $[8,9])$. Alternatively, naïve prey may recognize cues (particularly chemical cues) from novel predators if the predators have recently eaten prey that are of the same or similar species (reviewed in [10]).

The nine-banded armadillo (Dasypus novemcinctus) recently has experienced a relatively rapid period of range expansion [11]. Before the 1850s, the northern limit of the armadillo's range was northern Mexico, but the removal of natural barriers and the addition of roadways and railways allowed the armadillo to expand northward through Texas and into Southern Oklahoma and Arkansas; by the 1990s, the armadillo's range included most of Missouri and parts of Kansas and Nebraska [11]. In these regions armadillos occupy woodland, bottomland, brushland, and savannah habitats [12] where they are generalist foragers, apparently consuming any appropriately sized prey encountered during their searches of the leaf litter [13]. Stomach contents from a sample of armadillos in Arkansas revealed that, in addition to feeding on numerous taxa of invertebrates, they also consume some vertebrates, such as small reptiles and amphibians, including salamanders $[14,15]$.

Plethodontid (lungless) salamanders are especially diverse and abundant in deciduous forests in eastern 
North America. They may be particularly vulnerable to predation by armadillos because they are often active in leaf litter habitats at night (e.g., [16-18]) when armadillos are actively foraging [14]. Of particular concern are the smallbodied plethodontids, such as the Ozark zigzag salamander (Plethodon angusticlavius, $6.9-9.8 \mathrm{~cm}$ total length [19]), which are similar in size and shape to one of the armadillo's most commonly consumed prey, the earthworm [15].

For plethodontid salamanders, a primary mode for predator recognition is via chemical cues ("kairomones" [20]) left on the substrate by the predator. Predator recognition is typically tested by bioassays that determine whether responses of salamanders to predator kairomones are consistent with antipredator behavior. For example, stimuli from predatory garter snakes (Thamnophis sirtalis) and ringneck snakes (Diadophis punctatus) have elicited avoidance responses [21,22] and reduced foraging activity [23-25]; the latter behavior is consistent with both increased vigilance and decreased visibility to visual predators. Physiologically, antipredator responses can be manifested as increased respiration (e.g., [26]), apparently as a component of the frightor-flight response.

Salamanders retrieve chemical cues from the substrate (i.e., nonvolatile cues) using nasolabial cirri, and these cues are assessed via the vomeronasal organ; airborne (volatile) cues can be assessed using the main olfactory system [27]. Although salamanders experience predation from other mammals (skunks, raccoons, and shrews [28]), we are not aware of any studies that have investigated plethodontid salamander responses to mammal kairomones.

We tested whether Ozark zigzag salamanders from a population near the northern extent of the armadillo's expanded range would (1) respond to chemical stimuli from armadillos with behaviors that are consistent with an antipredator response and (2) distinguish between chemical cues from armadillos and a nonpredatory herbivorous mammal (white-tailed deer, Odocoileus virginianus). We performed a series of experiments where salamanders were exposed to either volatile or nonvolatile cues from armadillos, deer, and a blank control; we recorded both behavioral and metabolic responses.

\section{Methods}

2.1. Salamander Collection and Maintenance. We collected approximately 80 adult Ozark zigzag salamanders from sites in Taney County, MO, USA and White County, AR, USA during 2006-2009. Salamanders were housed in clear, plastic Petri dishes $(14.5 \mathrm{~cm}$ diameter, $1.5 \mathrm{~cm}$ depth) lined with moistened filter paper and kept inside an environmental chamber $\left(19^{\circ} \mathrm{C}, 12: 12\right.$ light: dark cycle). We fed salamanders a diet of 10-15 flies (Drosophila hydei) per week and changed filter paper biweekly.

2.2. Collection of Stimuli. We captured three armadillos with a landing net at Bull Shoals Field Station (BSFS) in Taney County, MO, USA. We placed the armadillos in large plastic containers $(55 \times 40 \times 30 \mathrm{~cm})$ at the field site and collected fecal matter (20-75 mL from each individual) when defecation occurred (within $60 \mathrm{~s}$ of capture). Armadillos were immediately released at the capture site after collection of feces. To serve as nonpredatory control stimuli, we also collected several pieces of moist deer scat $(50 \mathrm{~mL}$ total) in fields at BSFS. The scat was collected at dawn and estimated to be no more than 2-h old based upon the predawn activity of this species [29].

All fecal samples were diluted (9:1 dechlorinated tap water to fecal matter), homogenized with a blender, and passed through Aqua-Pure filter fiber to remove solid particles. The resulting liquid was divided into snap-cap tubes $(1.7 \mathrm{~mL}$ each) which were labeled and frozen. In addition to the predator and nonpredator treatments, we used dechlorinated tap water as a blank control. This control was prepared in the same manner as the other samples but without the addition of any fecal matter.

Experiment 1 (behavioral responses to substrate cues). We conducted Experiment 1 during October 2007. We fed each salamander 4-6 flies and changed its filter paper four days before each trial, providing individuals with sufficient time for pheromonal marking of the substrates [30]. We exposed salamanders $(n=23)$ individually to each of three stimuli (armadillo, deer, or blank water) in random order with four days between exposures.

Five min before each trial, we removed a salamander from its home Petri dish and placed it into a clean holding dish. We poured one of the three stimuli into the home Petri dish and rotated the dish to spread the stimulus evenly across the filter paper. Then we returned the salamander to its home dish which was surrounded by partitions on three sides to minimize visual disturbance. Trials began immediately after the salamander was introduced and lasted for $600 \mathrm{~s}$. Then an observer, who was blind to the treatment, recorded movement time (time spent walking), edge time (time spent with the head or body pressed against the sides of the Petri dish), and time in the flat posture (entire ventral surface in contact with substrate). Changes in activity and increased edge time have been reported as responses to predatory stimuli by salamanders in other studies (e.g., [31, 32]); flat behavior has not been reported in an antipredator context, but it is consistent with reduction of conspicuousness. After the completion of a trial, we moved each salamander to a clean Petri dish with new filter paper.

Experiment 2 (behavioral responses to airborne cues). In April 2009, we tested salamander responses to airborne stimuli. The stimuli were the same as in Experiment 1, but we exposed the salamanders differently. During each trial, we used a flow meter (Sable Systems, MFS2) to pump air (rate = $440 \mathrm{~mL}$ per $\mathrm{min}$ ) from a container holding a stimulus sample through a container that housed a salamander (Figure 1). The container holding the stimulus was a clean Petri dish with moistened filter paper, and the container holding the salamander was its home Petri dish. We observed the salamanders $(n=21)$ for $10 \mathrm{~min}$ and recorded the same behavioral patterns as in Experiment 1: movement time, edge time, and flat time. We exposed each salamander in 


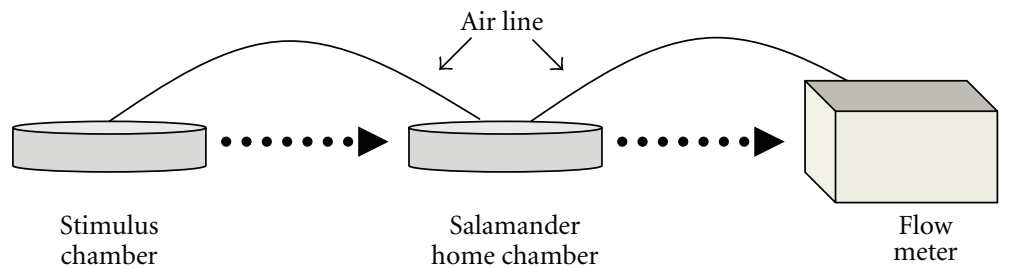

Figure 1: Diagram of the testing apparatus used to test behavioral responses of salamanders to airborne cues in Experiment 2 (diagram modified from Windel 2005). Salamanders occupied their home chambers which were connected by air line to stimulus chambers containing diluted armadillo feces, deer feces, or blank water; air was pumped from the stimulus chamber through the salamander's chamber via the flow meter. Dotted arrows indicate direction of flow.

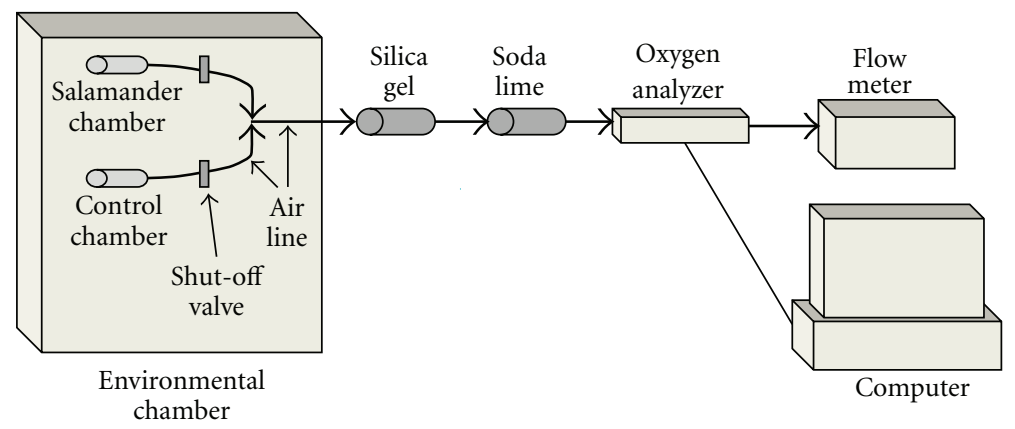

FIGURE 2: Apparatus used for testing oxygen consumption by salamanders exposed to substrate cues in Experiment 3 (diagram modified from Windel 2005). Air from a cylindrical chamber holding a salamander was pumped by the flow meter through an oxygen analyzer to measure oxygen consumption during a sealed-chamber period of $12 \mathrm{~min}$. Dark arrows indicate direction of flow.

a random order to each of the treatments, and the time between exposures was 6-7 days.

Experiment 3 (physiological responses to substrate cues). In May 2010, we recorded the oxygen consumption of salamanders exposed to each of the three treatments $(n=17$ per group). We placed salamanders in small chambers $(6 \mathrm{~mL}$ glass syringes); because salamanders frequently occupy earthworm or similar-sized burrows in nature [33], the small diameter of these tubes should not have represented a substantial stressor. The inside of the chamber was lined with filter paper that had been moistened with water from the salamander's home Petri dish. The chambers were kept inside an environmental chamber that maintained a temperature of $19^{\circ} \mathrm{C}$. Salamanders acclimated for $24 \mathrm{~h}$ in these chambers before trials began.

At the start of a trial, one of the three stimuli was selected and injected with a pipette onto the filter paper inside a chamber holding a salamander. The chamber was connected to an oxygen analyzer (Sable Systems, FC-10a; Figure 2) and then sealed for $12 \mathrm{~min}$. During this time, the oxygen analyzer recorded oxygen concentration levels from air that was being pumped (rate $=50 \mathrm{~mL}$ per $\mathrm{min}$ ) through a control chamber. The control chamber replicated the chamber holding the salamander except that it did not contain a salamander. After the $12-$ min period with the salamander's chamber sealed, we opened a valve that diverted air through the oxygen analyzer. After recording the oxygen concentration passing through the salamander's chamber for $10 \mathrm{~min}$, the valve was switched so that the analyzer received control air again. Before air reached the analyzer, it first passed through soda lime and silica gel to remove carbon dioxide and water, respectively. We analyzed the oxygen data using ExpeData Pro software, and we selected the proportional area tool to calculate the total amount of oxygen consumed during the same time frame for each individual's oxygen reading.

\section{Results}

Experiment 1 (behavioral responses to substrate cues). We performed general linear mixed models (GLMMs) with the experimental treatment as a fixed factor and subject as a random factor. Post hoc comparison tests were performed using Dunnett's method to compare the armadillo and deer treatments to the blank control. Only one salamander performed flat behavior, so no data are presented for this response variable.

Movement time differed significantly among treatments $(F=11.17, d f=2, P<0.001$; Figure 3(a)). Comparison tests revealed that movement time in the presence of the armadillo cues was significantly greater than it was in the blank control $(P<0.05)$, whereas movement time did not differ significantly between the deer and blank treatments $(P>0.05)$. The same pattern was found for edge time $(F=6.46, d f=2, P=0.003)$, salamanders exposed to armadillo cues spent more time on the edge than those in the blank treatment $(P<0.05)$, and there was no difference between edge time in the deer and blank treatments $(P<0.05$; Figure 3(b)). There also were consistent differences among individuals 


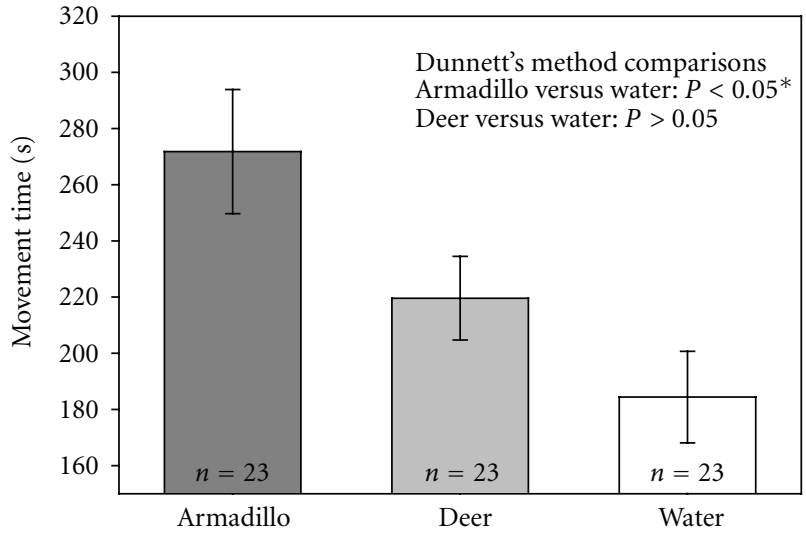

(a)

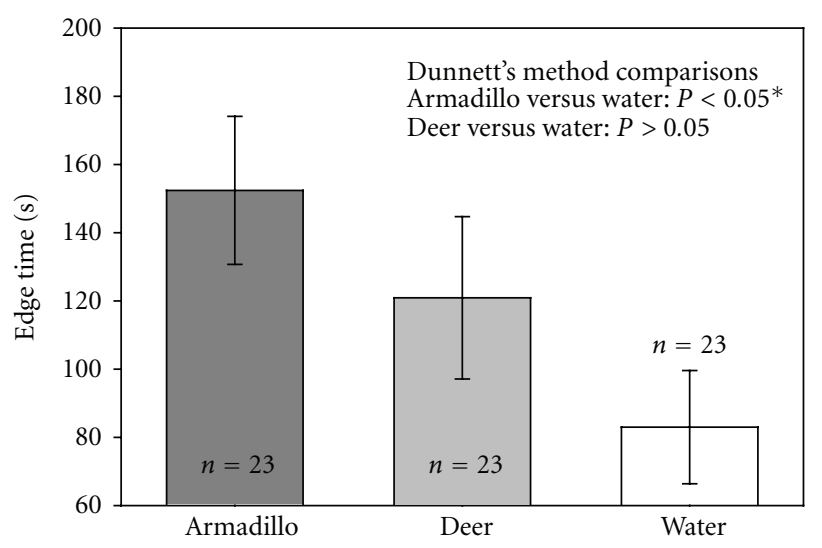

(b)

FIGURE 3: Experiment 1: behavioral responses to substrate cues. Time spent moving (a) and time spent on the edge of the arena (b) for salamanders exposed to stimuli from armadillos, deer, or blank control water. Bars and error bars represent means \pm 1 SE. Asterisks indicate statistical significance.

with respect to movement time $(F=3.64, d f=22, P<$ $0.001)$ and edge time $(F=5.04, d f=22, P<0.001)$.

Experiment 2 (behavioral responses to airborne cues). For Experiment 2, the GLMM analysis showed no statistical differences among the treatments for movement time $(F=$ 1.26, $d f=2, P=0.294$; Figure $4(\mathrm{a}))$ or edge time $(F=$ $1.24, d f=2, P=0.804)$, and no differences existed among individuals for these variables (movement time: $F=$ 0.99, $d f=22, P=0.497$; edge time: $F=1.03, d f=$ 22, $P=0.450)$. However, flat time was significantly different among individuals $(F=3.29, d f=22, P<0.001)$ and marginally different among treatments $(F=2.69, d f=$ 2, $P=0.080$; Figure $4(\mathrm{~b}))$.

Experiment 3 (physiological responses to substrate cues). The individuals in Experiment 3 were only tested once (i.e., independent groups), so we were unable to test for differences among individuals. To assess differences among treatments, we used a nonparametric Kruskal-Wallis ANOVA because the data were not normally distributed.



(a)

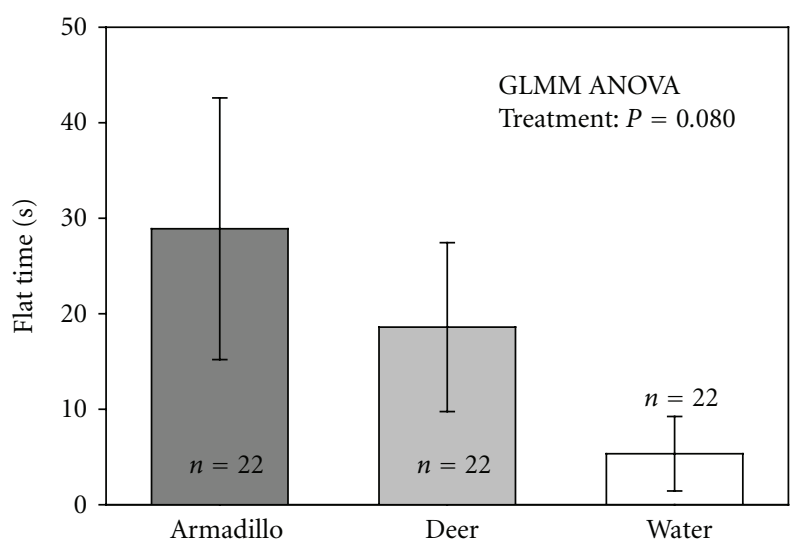

(b)

FIgURE 4: Experiment 2: behavioral responses to airborne cues. Time spent moving (a) and time spent in a flat posture (b) for salamanders exposed to stimuli from armadillos, deer, or blank control water. Bars and error bars represent means $\pm 1 \mathrm{SE}$.

Oxygen consumption is affected by body size, so we performed the analysis on the residuals of the oxygen data regressed against the mass of the salamanders after logtransforming both variables because of their curvilinear relationship in amphibians [34]. This statistical method was also used by Ligon and Peterson [35] for analyzing similar metabolic data in turtles. Statistical testing revealed a significant difference among the treatments $(H=6.06, P=$ 0.048; Figure 5). We then performed post hoc Dunnett's method comparisons, which indicated that, compared to the blank control, salamanders responded to armadillo stimuli with increased oxygen consumption $(P<0.05)$ but did not respond differently when exposed to deer stimuli $(P>0.05)$.

\section{Discussion}

Numerous species of plethodontid salamanders may be affected by the recent and future expansions of the armadillo's range. Thermal models, based on environmental criteria such as the annual number of freeze days, lead to the prediction that the armadillo will eventually move into the northeastern United States, including Appalachia [11] 


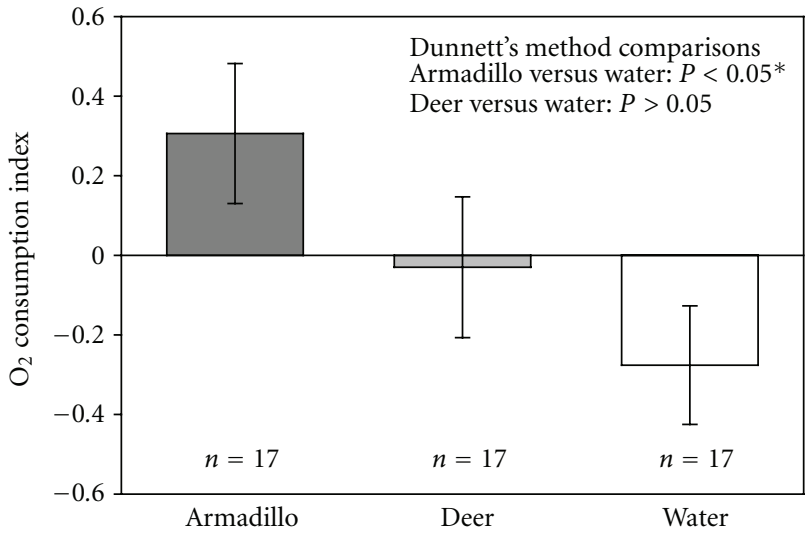

FIGURE 5: Experiment 3: physiological responses to substrate cues. Oxygen consumption index by salamanders exposed to stimuli from armadillos, deer, or blank control water in a closed chamber for $12 \mathrm{~min}$. Bars and error bars represent means $\pm 1 \mathrm{SE}$. The asterisk represents statistical significance. Index $=$ residuals of log-transformed oxygen consumption $(\mu \mathrm{L})$ regressed against logtransformed mass $(\mathrm{g})$.

where plethodontid salamander diversity is highest [36]. The Ozark zigzag salamander has lived sympatrically with armadillos for a relatively short period of time and, thus, provides a suitable model for understanding the effects, if any, that armadillo expansion may have on armadillo-naïve populations of salamanders.

Our data indicate that Ozark zigzag salamanders can discriminate between chemical stimuli from feces of armadillos (potential predators) and deer (nonpredators). In nature, feces are patchily distributed, so substrate cues would only be sporadically available as warning cues. We do not know the distance at which airborne cues from armadillo feces travel, but these cues might provide an earlier warning than substrate cues. Either airborne or substrate cues given off by the body of the armadillo, as opposed to feces, could provide a more efficient warning source, and future experiments should test whether these cues are detected by salamanders.

Interestingly, there also was an effect of individual subject on responses to the stimuli. Therefore, individuals that had relatively low-level responses to one cue tended to have relatively low-level responses to the other cues, and vice versa. Movement time (general activity) and edge time (a presumed escape activity) were individually consistent for substrate cues, and flat time was consistent for individuals for airborne cues. Similar individual differences in general activity and antipredator activity have been seen for a variety of species, including crabs (Heterozius rotundifrons) [37], European house crickets (Acheta domesticus) [38], and smallmouth bass (Micropterus dolomieu) [39]. Individual consistencies in behavior across more than one context ("behavioral syndromes") may have important implications for evolutionary ecology, including the maintenance of behavioral variation, the occurrence of behaviors that are apparently maladaptive, and characteristics of species such as the propensity to be invasive [40].
Overall, salamander responses to the armadillo cues were consistent with antipredator responses, but the nature of the response differed for volatile and nonvolatile cues. Salamanders responded to substrate cues from armadillos with increases in activity (Experiment 1), edge behavior (Experiment 1), and oxygen consumption (Experiment 3 ). Therefore, it appears that the primary antipredator strategy when substrate cues are detected is escape. Increased edge behavior has been reported for plethodontid salamanders in response to alarm/distress chemicals from conspecifics and has been interpreted as attempts to escape the chamber or to find crevices to serve as refuges [23,31]. Increases in general (nonforaging) activity have also been reported in response to snake kairomones (e.g., [41]). Numerous other species (e.g., $[42,43]$ ), including some salamanders (e.g., $[32,44])$, have shown the opposite responses of freezing or reduced activity, which presumably increases the probability that the prey will escape detection by visual predators. The tactic (flight versus freezing) chosen by prey is likely due in part to the type of foraging mode used by predators. Armadillos forage by digging or probing the ground with the nose [45], so freezing or reduced activity would be ineffective antipredator behaviors. Substrate cues may indicate that the armadillo is (or has recently been) in close proximity and so represents an immediate threat.

In contrast, salamanders exposed to airborne cues from armadillos did not increase their movement time or edge time, but instead spent more time performing the flat posture (Experiment 2). Although not statistically significant, activity tended to decrease in response to airborne cues from armadillos, which was opposite to the response to substrate cues in Experiment 1. Airborne cues may indicate that an armadillo is in the general vicinity, but not necessarily an immediate threat; in this case, flight might be a dangerous strategy that could result in the salamander drawing the predator's attention or bringing it into the predator's perceptual field. Alternatively, airborne cues may not provide sufficient information for salamanders to determine the precise nature of the threat. In any case, the response to airborne cues appears to be primarily a reduction in conspicuous behavior.

What is the mechanism by which Ozark zigzag salamanders discriminate between armadillo and deer stimuli? The salamanders may have detected armadillos specifically as a predator, perhaps through previous experience with armadillos, or they may have simply detected cues associated with the diet of the armadillo. Another small terrestrial salamander, the redback salamander ( $P$. cinereus), is known to be sensitive to dietary cues associated with snake predators $[41,46]$. Any predator that consumes invertebrate prey from the leaf litter is likely a threat to small terrestrial salamanders, so avoiding cues produced as metabolic byproducts of digestion of these prey is likely to be a good antipredator tactic. Furthermore, there may have been general chemical differences between feces from an herbivore such as deer and a carnivore such as armadillo. One potential way to address this question would be to test salamanders from more northeasterly populations (outside the range of armadillos). Because those salamanders would lack evolutionary history 
with armadillos, a response comparable to those in our experiments would suggest the recognition of diet-related cues, rather than to non-diet-related scents associated with the armadillo. Similarly, a lack of response by northeasterly populations would suggest that the responses we observed in our study were not solely based on diet cues. If the latter case is correct, populations of armadillo-naïve salamanders may face some initial vulnerability to armadillo expansion, but if so, it appears that the ability to recognize armadillo cues is either learned (e.g., [47]) or evolves (e.g., [48]) relatively quickly.

\section{Acknowledgments}

The authors thank Missouri State University and the Biology Department for support. This research was approved by Missouri State University's IACUC (protocol no. 2007M). Salamander collection was done under Arkansas Game and Fish permit no. 030120061 and Missouri Department of Conservation permit no. 13611. They are especially grateful to Nathan Windel who helped with several aspects of this work. They appreciate the use of Missouri State's Bull Shoals Field Station and thank the director, Janice Greene, and her staff. For assistance collecting salamanders or armadillos, they thank Jenny Parsons, Rob Hunt, and Amanda Crane, and, for technical assistance with analyzing oxygen data, they thank Day Ligon, Tom Tomasi, and Ty Sanders. They also thank Lynn Robbins for advice on catching armadillos.

\section{References}

[1] L. B. Kats and R. P. Ferrer, "Alien predators and amphibian declines: review of two decades of science and the transition to conservation," Diversity and Distributions, vol. 9, no. 2, pp. 99-110, 2003.

[2] M. Clavero and E. García-Berthou, "Invasive species are a leading cause of animal extinctions," Trends in Ecology and Evolution, vol. 20, no. 3, p. 110, 2005.

[3] K. G. Human and D. M. Gordon, "Exploitation and interference competition between the invasive Argentine ant, Linepithema humile, and native ant species," Oecologia, vol. 105, no. 3, pp. 405-412, 1996.

[4] J. Prenter, C. MacNeil, J. T. A. Dick, and A. M. Dunn, "Roles of parasites in animal invasions," Trends in Ecology and Evolution, vol. 19, no. 7, pp. 385-390, 2004.

[5] B. G. Gall and A. Mathis, "Innate predator recognition and the problem of introduced trout," Ethology, vol. 116, no. 1, pp. 47$58,2010$.

[6] D. L. Murray, J. D. Roth, and A. J. Wirsing, "Predation risk avoidance by terrestrial amphibians: the role of prey experience and vulnerability to native and exotic predators," Ethology, vol. 110, no. 8, pp. 635-647, 2004.

[7] V. T. Vredenburg, "Reversing introduced species effects: experimental removal of introduced fish leads to rapid recovery of a declining frog," Proceedings of the National Academy of Sciences of the United States of America, vol. 101, no. 20, pp. 7646-7650, 2004.

[8] M. C. O. Ferrari, A. Gonzalo, F. Messier, and D. P. Chivers, "Generalization of learned predator recognition: an experimental test and framework for future studies," Proceedings of the Royal Society B, vol. 274, no. 1620, pp. 1853-1859, 2007.
[9] A. Sih, D. I. Bolnick, B. Luttbeg et al., "Predator-prey naïveté, antipredator behavior, and the ecology of predator invasions," Oikos, vol. 119, no. 4, pp. 610-621, 2010.

[10] R. Hunt, Behavioral and metabolic responses of juvenile spotted salamanders (Ambystoma maculatum) to alarm cues from damaged skin and predator diet, thesis, Missouri State University, 2011.

[11] J. F. Taulman and L. W. Robbins, "Recent range expansion and distributional limits of the nine-banded armadillo (Dasypus novemcinctus) in the United States," Journal of Biogeography, vol. 23, no. 5, pp. 635-648, 1996.

[12] J. K. Jones, D. M. Armstrong, R. S. Hoffmann, and C. J. Jones, Mammals of the Northern Great Plains, University of Nebraska Press, Lincoln, Neb, USA, 1983.

[13] K. K. Smith and K. H. Redford, "The anatomy and function of the feeding apparatus in two armadillos (Dasypoda): anatomy is not destiny," Journal of Zoology, vol. 222, no. 1, pp. 27-47, 1990.

[14] H. S. Fitch, P. Goodrum, and C. Newman, "The armadillo in the southeastern United States," Journal Of Mammalogy, vol. 33, pp. 21-37, 1952.

[15] R. S. Sikes, G. A. Heidt, and D. A. Elrod, "Seasonal diets of the nine-banded armadillo (Dasypus novemcinctus) in a northern part of its range," American Midland Naturalist, vol. 123, no. 2, pp. 383-389, 1990.

[16] H. Heatwole, "Environmental factors influencing local distribution and activity of the salamander, Plethodon cinereus," Ecology, vol. 43, pp. 460-472, 1962.

[17] C. L. Ralph, "A diurnal activity rhythm in Plethodon cinereus and its modification by an influence having a lunar frequency," The Biological Bulletin, vol. 113, pp. 188-197, 1957.

[18] M. C. Grover, "Influence of cover and moisture on abundances of the terrestrial salamanders Plethodon cinereus and Plethodon glutinosus," Journal of Herpetology, vol. 32, no. 4, pp. 489-497, 1998.

[19] S. E. Trauth, H. W. Robinson, and M. V. Plummer, The Amphibians and Reptiles of Arkansas, University of Arkansas Press, Fayetteville, Ark, USA, 2004.

[20] W. L. Brown, T. Eisner, and R. J. Whittaker, "Allomones and kairomones: transpecies chemical messengers," BioScience, vol. 20, pp. 21-22, 1971.

[21] J. D. McDarby, D. M. Madison, and J. C. Maerz, "Chemosensory avoidance of predators by the red-backed salamander, Plethodon cinereus," in Advances in Chemical Signals in Vertebrates, R. E. Johnston, D. Muller-Schwarze, and P. Sorensen, Eds., pp. 489-495, Kluwer Academic/Plenum, New York, NY, USA, 1999.

[22] A. M. Sullivan, J. C. Maerz, and D. M. Madison, "Anti-predator response of red-backed salamanders (Plethodon cinereus) to chemical cues from garter snakes (Thamnophis sirtalis): laboratory and field experiments," Behavioral Ecology and Sociobiology, vol. 51, no. 3, pp. 227-233, 2002.

[23] R. T. Watson, A. Mathis, and R. Thompson, "Influence of physical stress, distress cues, and predator kairomones on the foraging behavior of Ozark zigzag salamanders, Plethodon angusticlavius," Behavioural Processes, vol. 65, no. 2, pp. 201-209, 2004.

[24] J. C. Maerz, N. L. Panebianco, and D. M. Madison, "Effects of predator chemical cues and behavioral biorhythms on foraging activity of terrestrial salamanders," Journal of Chemical Ecology, vol. 27, no. 7, pp. 1333-1344, 2001.

[25] N. L. Windel, Behavioral and metabolic responses of the southern red-backed salamander (Plethodon serratus) to predatory 
stimuli: influence of body size, thesis, Missouri State University, 2005.

[26] L. A. Hawkins, J. D. Armstrong, and A. E. Magurran, "Predator-induced hyperventilation in wild and hatchery Atlantic salmon fry," Journal of Fish Biology, vol. 65, pp. 88100, 2004.

[27] E. M. Dawley and A. H. Bass, "Chemical access to the vomeronaal organs of a plethodontid salamander," Journal of Morphology, vol. 200, no. 2, pp. 163-174, 1989.

[28] K. D. Wells, The Ecology and Behavior of Amphibians, The University of Chicago Press, Chicago, Ill, USA, 2007.

[29] G. G. Montgomery, "Nocturnal movements and activity rhythms of white-tailed deer," Journal of Wildlife Management, vol. 27, pp. 422-427, 1963.

[30] A. Mathis and E. Britzke, "The roles of body size and experience in agonistic displays of the ozark zigzag salamander, Plethodon angusticlavius," Herpetologica, vol. 55, no. 3, pp. 344-352, 1999.

[31] A. Mathis and D. Lancaster, "Response of terrestrial salamanders to chemical stimuli from distressed conspecifics," Amphibia-Reptilia, vol. 19, pp. 330-335, 1998.

[32] A. Mathis, K. L. Murray, and C. R. Hickman, "Do experience and body size play a role in responses of larval ringed salamanders, Ambystoma annulatum, to predator kairomones? Laboratory and field assays," Ethology, vol. 109, no. 2, pp. 159 170, 2003

[33] T. S. Ransom, "Earthworms, as ecosystem engineers, influence multiple aspects of a salamander's ecology," Oecologia, vol. 165, no. 3, pp. 745-754, 2011.

[34] W. E. Duellman and L. Trueb, Biology of Amphibians, McGraw Hill, New York, NY, USA, 1986.

[35] D. B. Ligon and C. C. Peterson, "Physiological and behavioral variation in estivation among mud turtles (Kinosternon spp.)," Physiological and Biochemical Zoology, vol. 75, no. 3, pp. 283293, 2002.

[36] A. R. Kiester, "Species density of North American amphibians and reptiles," Systematic Zoology, vol. 20, pp. 127-137, 1971.

[37] B. A. Hazlett and C. E. Bach, "Individuality in the predator defense behaviour of the crab Heterozius rotundifrons," Behaviour, vol. 147, no. 5-6, pp. 587-597, 2010.

[38] A. D. M. Wilson, E. M. Whattam, R. Bennett, L. Visanuvimol, C. Lauzon, and S. M. Bertram, "Behavioral correlations across activity, mating, exploration, aggression, and antipredator contexts in the European house cricket, Acheta domesticus," Behavioral Ecology and Sociobiology, vol. 64, no. 5, pp. 703715, 2010.

[39] K. L. Smith, J. G. Miner, D. D. Wiegmann, and S. P. Newman, "Individual differences in exploratory and antipredator behaviour in juvenile smallmouth bass (Micropterus dolomieu)," Behaviour, vol. 146, no. 2, pp. 283-294, 2009.

[40] A. Sih, A. Bell, and J. C. Johnson, "Behavioral syndromes: an ecological and evolutionary overview," Trends in Ecology and Evolution, vol. 19, no. 7, pp. 372-378, 2004.

[41] A. M. Sullivan, D. M. Madison, and J. R. Rohr, "Variation in the antipredator responses of three sympatric plethodontid salamanders to predator-diet cues," Herpetologica, vol. 60, no. 4, pp. 401-408, 2004.

[42] D. P. Chivers and R. J. F. Smith, "Chemical alarm signalling in aquatic predator-prey systems: a review and prospectus," Ecoscience, vol. 5, no. 3, pp. 338-352, 1998.
[43] M. E. Fraker, "The dynamics of predation risk assessment: responses of anuran larvae to chemical cues of predators," Journal of Animal Ecology, vol. 77, no. 4, pp. 638-645, 2008.

[44] K. J. Epp and C. R. Gabor, "Innate and learned predator recognition mediated by chemical signals in Eurycea nana," Ethology, vol. 114, no. 6, pp. 607-615, 2008.

[45] C. M. McDonough, "Pairing behavior of the nine-banded armadillo (Dasypus novemcinctus)," American Midland Naturalist, vol. 138, no. 2, pp. 290-298, 1997.

[46] D. L. Murray and C. L. Jenkins, "Perceived predation risk as a function of predator dietary cues in terrestrial salamanders," Animal Behaviour, vol. 57, no. 1, pp. 33-39, 1999.

[47] G. E. Brown, D. P. Chivers, and R. Jan F Smith, "Differential learning rates of chemical versus visual cues of a northern pike by fathead minnows in a natural habitat," Environmental Biology of Fishes, vol. 49, no. 1, pp. 89-96, 1997.

[48] P. Berthold, A. J. Helbig, G. Mohr, and U. Querner, "Rapid microevolution of migratory behaviour in a wild bird species," Nature, vol. 360, no. 6405, pp. 668-670, 1992. 

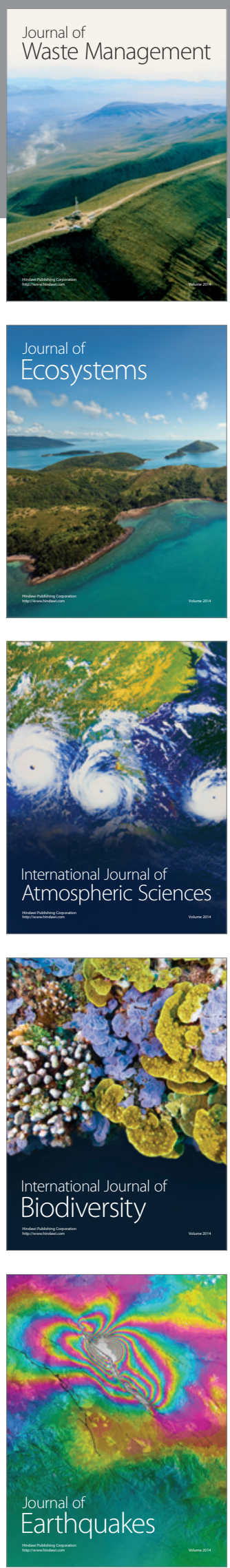
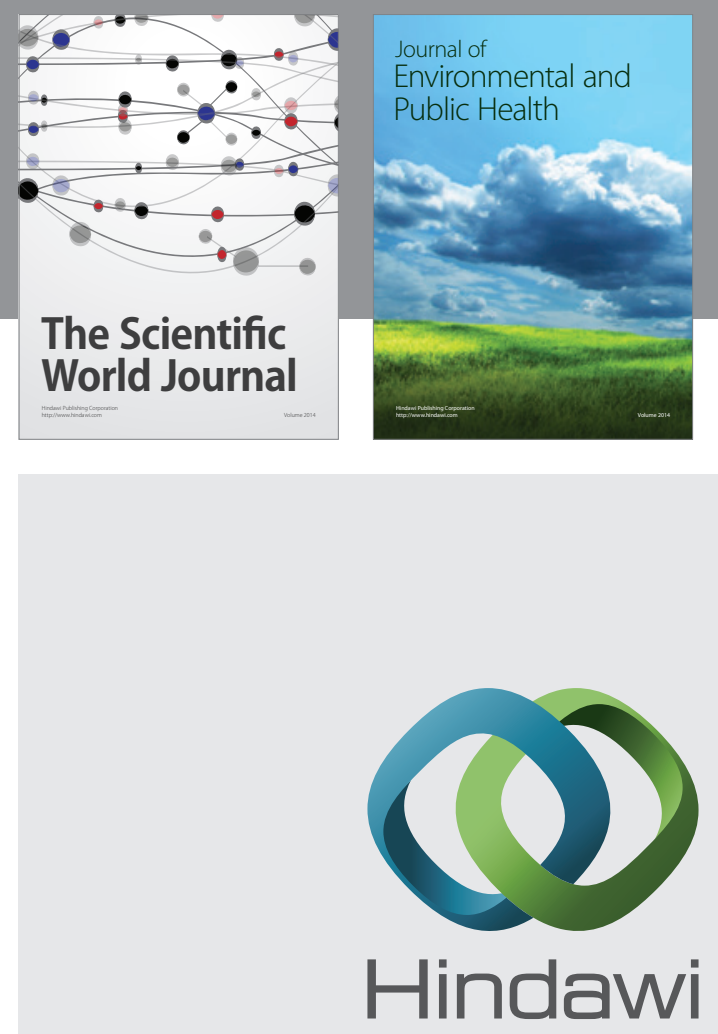

Submit your manuscripts at

http://www.hindawi.com
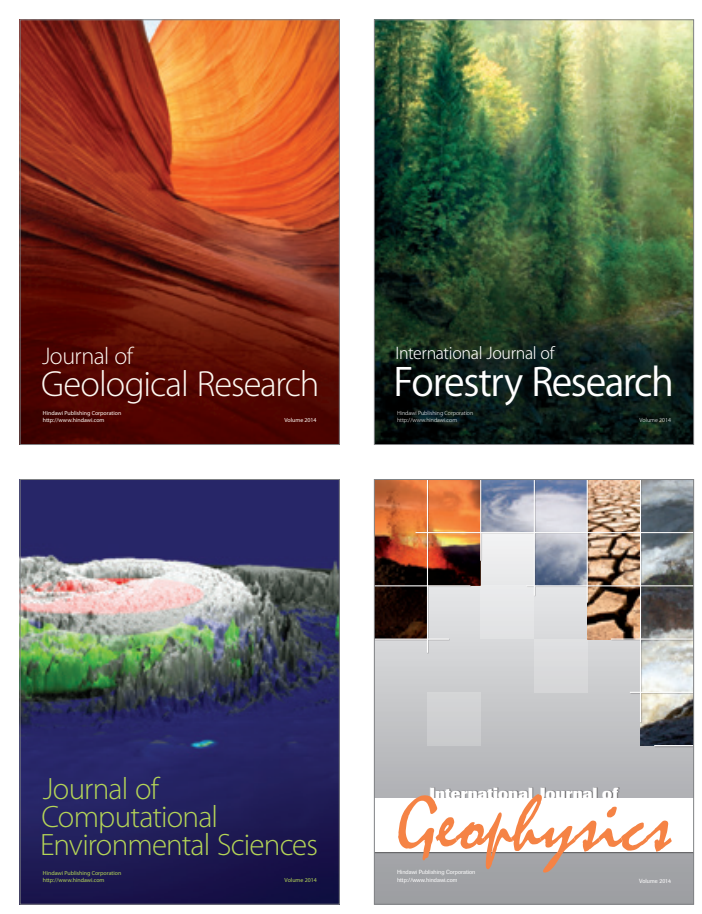
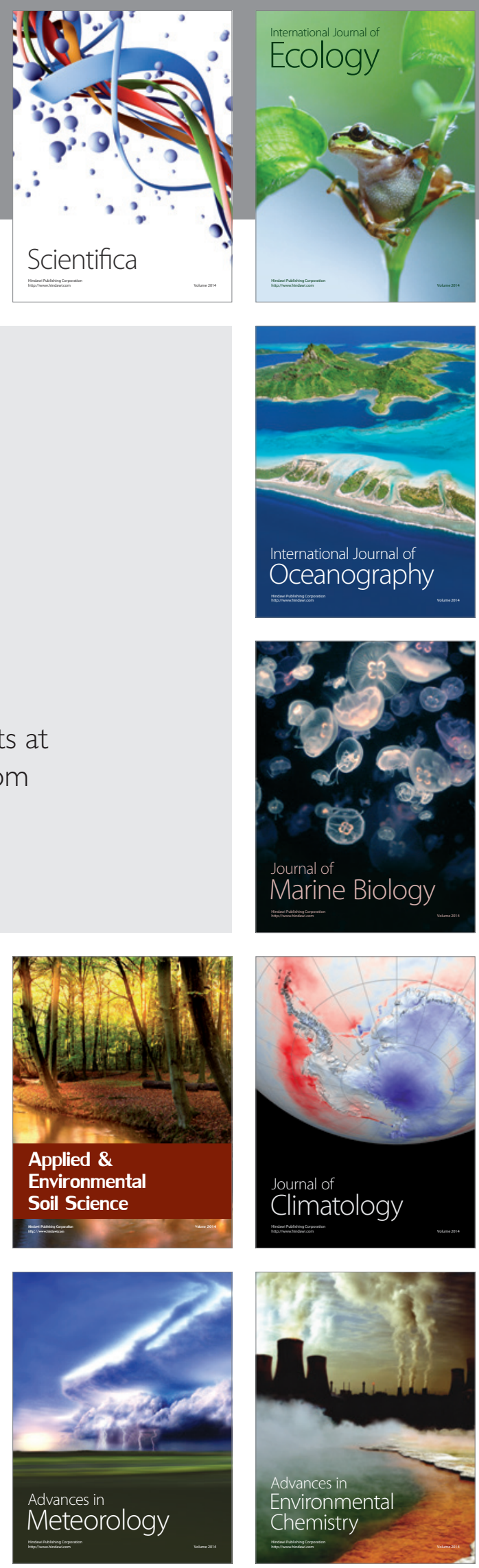